AN. MED. INTERNA (Madrid) Vol. 21, N. $^{\circ} 4$, pp. $183-184,2004$

\title{
Distrofia simpático refleja de extremidades inferiores, politópica y recurrente en dos hermanos
}

\author{
C. BRUSCAS IZU, C.H. BELTRÁN AUDERÁ, F. JIMÉNEZ ZORZO \\ Unidad de Reumatología. Hospital Miguel Servet. Zaragoza
}

REFLEX SYMPATHETIC DYSTROPHY IN LOWER LIMBS, POLYTOPIC AND RECURRENT IN TWO BROTHERS

\begin{abstract}
RESUMEN
La distrofia simpático refleja (DSR) puede estar relacionada con diferentes factores desencadenantes o predisponentes. No obstante, en la literatura médica no hay muchas publicaciones referentes a un terreno genético o familiar en la DSR. Por ello, presentamos el caso de 2 hermanos con similar HLA y con DSR de extremidades inferiores, politópica y recurrente.
\end{abstract}

PALABRAS CLAVE: Distrofia simpático refleja. Algodistrofia. Familiar. Genética. Politópica. Recurrent.

\begin{abstract}
Reflex sympathetic dystrophy (RSD) has been related to a variety of inciting and predisposing factors. However, there are few reports of a familiar or genetic background in RSD. This paper describes two cases of RSD polytopic and recurrent in lower limbs of two brothers with similar HLA.
\end{abstract}

KEY WORDS: Reflex sympathetic dystrophy. Algodystrophy. Familiar. Genetic. Polytopic. Recurrent.

Bruscas Izu C, Beltrán Audera CH, Jiménez. Zorzo F. Distrofia simpático refleja de extremidades inferiores, politópica y recurrente en dos hermanos. An Med Interna (Madrid) 2004; 21: 183-184.

\section{INTRODUCCIÓN}

La distrofia simpático refleja (DSR) es una entidad clínica que suele acompañarse de dolor, inflamación, cambios vasomotores, hiperestesia e impotencia funcional; evolucionando, en ocasiones, hacia cambios tróficos. Su mecanismo etiopatogénico no es bien conocido y en ella se reconocen distintos factores desencadenantes y predisponentes de la misma; si bien, en algunos casos no se encuentra una causa aparente (1). Entre los factores desencadenantes, el traumatismo es el más frecuente y, en menor medida, se involucran otros. Por otra parte, se consideran factores predisponentes la diabetes, la hipertrigliceridemia y un terreno psicológico alterado $(2,3)$. No obstante, en la literatura médica no es frecuente encontrar trabajos que hagan referencia a formas familiares o a un posible terreno genético predisponente. Por ello, presentamos los casos poco habituales de dos hermanos, con idéntico HLA y con DSR de extremidades infe- riores, politópicas, refractarias al tratamiento, de larga evolución y recurrentes, y discutimos sobre la posible implicación genética en esta afección.

\section{CASOS APORTADOS}

Caso 1. Varón de 47 años de edad que en enero de 1998 comenzó con dolor persistente, inflamación e impotencia funcional en la rodilla izquierda, tras lo que describió el paciente como un mal giro de dicha rodilla. En la radiología convencional y en la tomografía de esta rodilla se apreciaba una desmineralización, sin otros datos relevantes. Además, en la gammagrafía ósea había una captación intensa en la rodilla izquierda. Se le solicitó una RMN en la que se objetivaba edema de la meseta tibial medial y pequeña colección líquida intraarticular. El estudio analítico habitual (VSG, hemograma y bioquímica general) no aportaba datos de interés. El paciente fue tratado con AINE sin buen resultado. A los 5 meses de evolución fue visto en nuestras consultas por sintoma-

Trabajo aceptado: 20 de octubre de 2003

Correspondencia: Carlos Bruscas Izu. Avda. Goya, 75-77, 4º izqda. 50005 Zaragoza 
tología añadida de dolor y tumefacción global del pie y tobillo izquierdos, con desmineralización radiológica. El cuadro clínico se etiquetó de DSR y se solicitó un HLA, al presentar su hermana una situación parecida, con resultado de: A2, B44, B62, BW4, BW6, DRB1*01, DRB1*11, DRB4*, DQB1*03(8), DQB1*05. Se comenzó tratamiento con reposo relativo, corticoides a dosis bajas (10 mg. de prednisona, disminuyendo progresivamente), calcitonina y rehabilitación, con poca mejoría. Posteriormente, en la Unidad del Dolor se añadió amitriptilina, tramadol y clonazepan, con práctica resolución de la sintomatología de la rodilla y con mejoría lenta del tobillo y pie. Al año de evolución, presentó recidiva clínica en la rodilla izquierda y unos meses más tarde similares síntomas en la rodilla derecha, siendo entonces tratado con gabapentina y obteniendo una insuficiente respuesta. Un año después de iniciar la clínica de la rodilla derecha y persistiendo todavía dolor e impotencia funcional, se añadía clínica de dolor, edema e impotencia funcional del tobillo derecho. Actualmente, tras 3 años de evolución, persiste sintomatología del tobillo derecho y, en menor grado, de la rodilla derecha.

Caso 2. Mujer de 43 años de edad, hermana del anterior, con antecedente personal de depresión y DSR (diagnosticada y tratada en otro Servicio). En este sentido y según consta en informes, tuvo una DSR del pie y tobillo izquierdos, a los 2 meses de una contusión sobre el pie izquierdo, que fue tratada con AINE, corticoides, calcitonina, magnetoterapia y rehabilitación evolucionando de manera insatisfactoria hacia una fase fría con alteraciones tróficas, a los 6 meses del inicio. Ante la mala evolución, se decidió a los 9 meses tratamiento con fentolamina, resolviéndose la situación. Al mismo tiempo, apareció una DSR en la rodilla izquierda, también con mala respuesta al tratamiento médico que motivó, a los 3 meses de evolución, bloqueos endovenosos con guanetidina ( 3 en total), desapareciendo toda la sintomatología en 2 meses. No obstante, en este momento y de forma aditiva, comenzó con dolor, edema, alteraciones vasomotoras, hipersudoración e impotencia funcional en el pie y tobillo derechos, acudiendo a nuestro Servicio. En la exploración física se constataron signos inflamatorios y limitación de la movilidad. Por otra parte, en el estudio analítico no se apreciaron parámetros inflamatorios y el HLA era idéntico al de su hermano. En la gamamgrafía en tres fases se evidenció un incremento de la perfusión y permeabilidad capilar en la fase vascular y una hipercaptación en la fase ósea en dichas zonas. De otro modo, en la RMN se informó de la presencia de una hiposeñal en T1 y de una hiperseñal en T2, descartándose una osteonecrosis. Por tanto, la paciente fue diagnosticada de DSR y puesta en tratamiento con reposo relativo, prednisona a dosis bajas, calcitonina, amitripitilina y rehabilitación, con mejoría paulatina a lo largo de los meses. En la actualidad, con algo más de 2 años de evolución, presenta molestias discretas y no incapacitantes en pie y tobillo derechos.

\section{DISCUSIÓN}

Son pocos los trabajos encontrados en la literatura médica que hagan referencia a casos familiares o a la predisposición genética en la DSR. En este sentido, Albert (4) aludió en su artículo a tres hermanos afectados de una DSR de la cadera, de aparición espontánea y recurrente al cabo del tiempo en la cadera contralateral en los tres casos. Esta presentación familiar sugería al autor una posible predisposición genética, suposición avalada por el hallazgo de un HLA idéntico entre ellos. En nuestro caso, los dos hermanos también presentan el mismo HLA, aunque diferente al del trabajo precedente, y la DSR también fue recurrente en otras articulaciones.

Más tarde, Greipp (5) presentó 3 familias afectadas de una DSR y sugirió la inclusión del estudio genético entre las pruebas diagnósticas de esta entidad. En esta línea, Erdmann (6) comentaba 2 casos familiares de DSR de extremidades superiores que requirieron posterior amputación. El autor planteaba que cierta personalidad y factores familiares pudieran influir en un mal pronóstico, pero no hizo mención ni estudió el HLA.

Mailis (7) determinó en 1994 el HLA clase I y II en una población de 15 mujeres de raza caucásica afectadas de una DSR, encontrando una asociación con el HLA A3, B7 y DR2 (15) y siendo el primer estudio que relacionó la presencia del DR2 (15) con la mala respuesta al tratamiento. De este modo, nuestros dos pacientes tienen un HLA idéntico pero no el considerado por Mailis de riesgo para la aparición de una DSR ni el de supuesta resistencia al tratamiento. El trabajo de Mailis recogía en su serie dos casos, catalogados como espontáneos y politópicos, que presentaban el HLA DR1 y, además, compartían con los nuestros ciertas características clínicas como la aparición tras un traumatismo mínimo e incluso inexistente, la mayor afectación de extremidades inferiores, su mayor duración y la refractariedad al tratamiento.

Por último, Kemler (8) también determinó el HLA clase I y II en una población de 52 DSR y encontró un aumento significativo del HLA DQ1 respecto al grupo control.

En conclusión, el hecho de haber encontrado en dos hermanos, con un HLA idéntico, una DSR de similares características, nos sugiere considerar, como a otros autores, que pudiera haber un terreno genético predisponente de la DSR. En este sentido, todos los trabajos mencionados en la discusión presentan casuísticas pequeñas y, por tanto, serán necesarios trabajos más amplios que incluyan el HLA en el estudio analítico de la DSR, para confirmar esta sugerencia.

\section{Bibliografía}

1. Bruscas C, Medrano M, Simon L. El diagnóstico de la distrofia simpático refleja. An Med Interna (Madrid) 1999; 16: 308-14.

2. Bruscas C, Simón L. Algodistrofia: terreno favorecedor y factores desencadenantes. An Med Interna (Madrid) 1992; 9: 401-5.

3. Bruscas Izu C, Pérez Echeverría MJ, Medrano San Ildefonso M, Hijos S, Simon L. Distrofia simpático refleja: Aspectos psicológicos y psicopatológicos. An Med Interna (Madrid) 2001; 18: 38-44.

4. Albert J, Ott H. Three brothers with algodystrophy of the hip. Ann Rheum Dis 1983; 42: 411-24.

5. Greipp ME, Thomas AF: Familial occurences reflex sympathetic dys- trophy. Clin J Pain 1991; 7: 48.

6. Erdmann MWH, Wynn-Jones CH. Familial reflex sympathetic dystrophy syndrome and amputation. The Brittish Journal of Accident Surgery 1992; 23: 136-8.

7. Mailis A, Wade J. Profile of caucasian women with possible genetic predisposition to reflex sympathetic dystrophy: a pilot study. Clin J Pain 1994; 10: 210-7.

8. Kemler MA, Van de Vusse AC, Van den Berg-Loonen EM et al. HLADQ1 associated with reflex sympathetic dystrophy. Neurology 1999; 53: 1350-1. 